\title{
Granulomatosis after autologous stem cell transplantation in nonHodgkin lymphoma - experience of single institution and a review of literature
}

\author{
Lucka Boltezar $^{1}$, Ivana Zagar², Barbara Jezersek Novakovic ${ }^{1}$ \\ ${ }^{1}$ Division of Medical Oncology, Institute of Oncology Ljubljana, Ljubljana, Slovenia \\ ${ }^{2}$ Department of Nuclear Medicine, Institute of Oncology Ljubljana, Ljubljana, Slovenia
}

Radiol Oncol 2016; 50(4): 355-359.

Received 21 May 2015

Accepted 30 June 2015

Correspondence to: Assoc. Prof. Barbara Jezeršek Novaković, M.D., Ph.D., Division of Medical Oncology, Institute of Oncology Ljubljana, Zaloška 2, 1000 Ljubljana, Slovenia. Phone: +386 1587 9280; Fax: +38615879305; E-mail: bjezersek@onko-i.si

Disclosure: No potential conflicts of interest were disclosed.

Background. Sarcoidosis before and after treatment of malignancy is an important differential diagnosis that has to be distinguished from lymphoma.

Patients and methods. Hodgkin lymphoma, diffuse large B-cell lymphoma and aggressive follicular lymphoma are being staged and treatment effect is evaluated with PET-CT. We report three cases of aggressive lymphoma after high dose therapy and autologous stem cell transplantation with positive lymph nodes on PET-CT, which were histologically diagnosed as sarcoidosis/granulomatosis. In the literature, we found that false positive lymph nodes were more common after allogeneic than after autologous transplantation.

Conclusions. Post-treatment PET-CT positive lymph nodes should always be examined histologically prior to any further treatment decision to avoid unnecessary toxic procedures.

Key words: granulomatosis; nonHodgkin lymphoma; PET-CT; differential diagnosis; tissue biopsy

\section{Introduction}

Sarcoidosis is a multisystemic disease of so far unknown cause. It is characterised by CD4+ T cell alveolitis, followed by formation of noncaseating immune granulomas in involved organs, mainly the lungs and the lymphatic system. Mortality is higher in patients with sarcoidosis than in general population, pulmonary fibrosis being the main cause of death. Systemic corticosteroids are the main treatment. ${ }^{1}$ Sarcoidosis has been linked to malignancy in various aspects. Usually it precedes malignancy, less frequently is it diagnosed after the initial treatment of cancer. Pathogenesis of sarcoidosis after treatment of malignancy is still unknown but it can be a misleading finding especially in lym- phoma patients. ${ }^{2}$ Positron emission tomographic (PET) scanning using 18F-fluorodeoxyglucose (18F-FDG) combined with the computer tomography (CT) is used for initial diagnosis of lymphoma and for later follow-up examinations, especially in Hodgkin lymphoma, diffuse large B-cell lymphoma and aggressive follicular lymphoma., Still, positive post-treatment scans should be interpreted with caution. Positive lymph nodes in this setting are namely highly suspicious of a lymphoma relapse. ${ }^{5-8}$ Nonetheless, further treatment without biopsy of the suspicious sites could have detrimental consequences for the patient, starting with unnecessary conventional chemotherapy and proceeding to even more toxic allogeneic stem cell transplantation. 


\section{PET-CT imaging and evaluation}

Numerous studies have documented that PET scanning detects actively metabolizing tumor cells in residual masses of FDG-avid lymphomas following chemotherapy. It was shown that the persistent abnormal uptake is a predictor of early relapse or poor survival., ${ }^{9} 10$ After completion of the treatment, the CT scan of patients with nonHodgkin lymphomas (NHL) may show evident residual mass which can be either fibrosis or non-viable tumour. Yet, the PET scanning of these sites or combined PET-CT scanning appears to differentiate quite well between the relapse and the remission in most cases. ${ }^{5,11}$ Even though, post-therapy PET positivity requires tissue biopsy or further evaluation $^{5-8}$, same as the mid-therapy PET positivity. ${ }^{8}$ An important supplementation of the PET scan with the CT imaging is to identify the site for optimal sampling of the tissue therefore defining the most appropriate invasive procedure - in all our cases the bronchoscopy with biopsy - or to define the morphology of the extranodal tissue which was positive on PET-CT. ${ }^{8}$

In 2014, the new consensus of the international conference on malignant lymphoma imaging work group was published. ${ }^{3}$ It includes recommendations on the use of PET-CT scanning using 18F-FDG for the assessment of treatment of Hodgkin lymphoma, diffuse large B-cell lymphoma and aggressive follicular lymphoma. For primary mediastinal

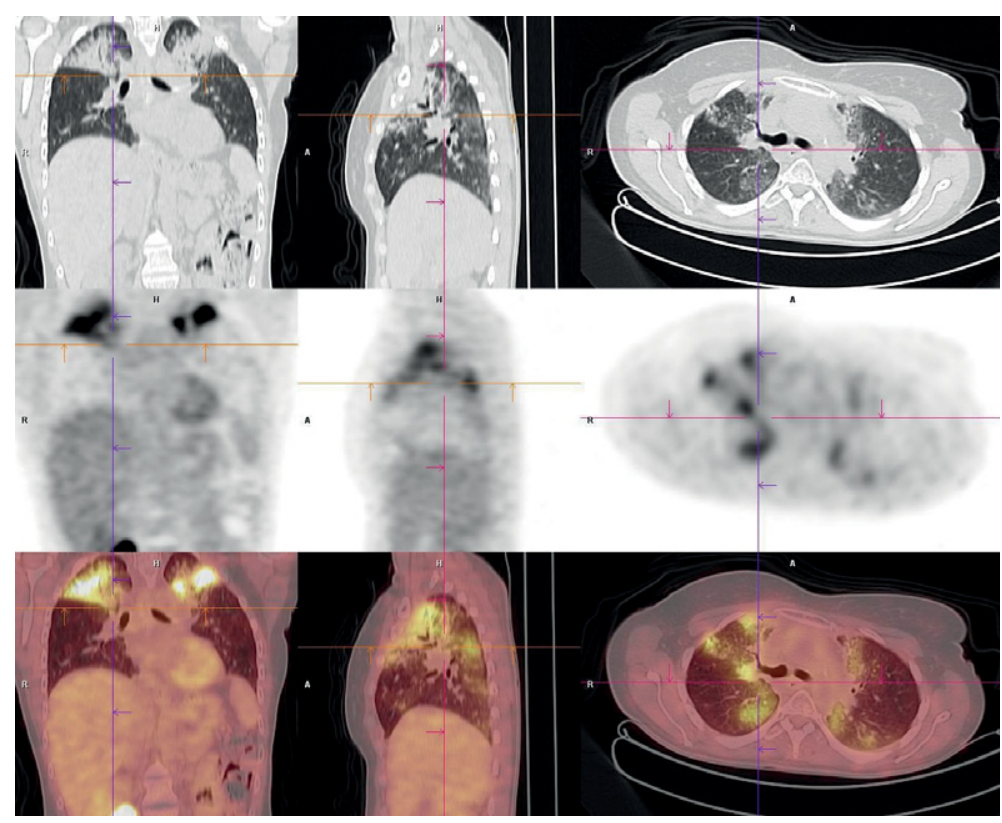

FIGURE 1. PET-CT patient1: Suspicious relapse in pulmonal tissue. large B-cell lymphoma the routine usage of PETCT scan is not validated and further prospective studies are warranted. ${ }^{3}$ Still, the primary mediastinal lymphomas are like the diffuse large B cell lymphomas aggressive lymphomas with high proliferation rate and the PET-CT should supposedly be an adequate diagnostic procedure both for staging and response evaluation purposes.

\section{Our patients after autologous stem cell transplantation}

In our observation through past few years, we found three different cases of pulmonary granulomatosis and sarcoidosis in lymphoma patients. All three patients were young females, aged between 33 and 40 years. Two of them had the primary mediastinal large B cell lymphoma and one had the diffuse large B-cell lymphoma. All three received standard first line immune-chemotherapy, relapsed and then following conventional salvage treatment underwent high dose treatment and autologous stem cell transplantation (AuSCT). All three had the control PET-CT scan 4-6 months following AuSCT (Figure 1, 2 and 3). Patient in Figure 1 had suspicious infiltrates in the lungs, paramediastinally and in the right pulmonary hilus. The thoracic $X$-ray was normal. The transbronchial biopsy showed granulomatosis and with bronchoalveolar lavage lymphocytic alveolitis with abnormal CD4/ CD8 ratio was discovered. Therefore, the diagnosis of sarcoidosis was set and the patient initiated the corticosteroid therapy. Patient in Figure 2 had suspicious infiltrates on PET-CT paratracheally, below the carina, close to the aortic arcus, between the liver and thoracic wall, under the diaphragm, in the spleen, some lesions in the liver and in the mesenterium, nodular lesions in the lungs and in the left and right pulmonary hili. The X-ray revealed enlarged mediastinum due to the lymph nodes. The transbronchial biopsy showed granulomatosis and the bronchoalveolar lavage discovered lymphocytic alveolitis with abnormal CD4/CD8 ratio. A normal serum level of calcium and angiotensin converting enzyme was found in the blood. The corticosteroid therapy was initiated. Patient in Figure 3 had an enlarged mediastinum on the $X$-ray of the thorax and suspicious infiltrates on PET-CT in the neck region, paratracheally, behind the aortic arc, in the right and left pulmonary hili, subcarinally, between the pancreas and the stomach and beside the hepatic artery and inferior vena cava. The transbronchial biopsy showed granulomatose changes, yet alveolitis was not present and 
the serum concentrations of calcium and angiotensin converting enzyme were normal. The patient was observed and the corticosteroid therapy has not been initiated. At present, all three patients are under regular control and none of them relapsed with lymphoma for the second time between 6 and 46 months following AuSCT. None of our patients complained of any symptoms of systemic sarcoidosis like the two cases described below. ${ }^{12,13}$

All three patients were immunocompromised following the stem cells transplantation. Lymphadenopathy in immunocompromised patients may be observed with acute viral infections (Cytomegalovirus, Ebstein-Barr virus), sarcoidosis, and infections due to mycobacteria, Cryptococcus spp., and with some drug reactions (e.g. trimethoprim-sulfamethoxazole). ${ }^{14}$

\section{Sarcoidosis and malignancies}

In the 1960s, a higher incidence of cancer was observed in sarcoidosis patients. Lung cancer occurred 3 times and malignant lymphoma 11.5 times more frequently than in control population. ${ }^{15}$ In 1986, Brincker introduced a new condition called the sarcoidosis-lymphoma-syndrome. ${ }^{16}$ Most of the patients with this syndrome had chronic, active sarcoidosis and later developed the lymphoproliferative malignancy. The most frequently observed was Hodgkin lymphoma, yet NHL, chronic lymphocytic leukaemia and paraproteinaemia were also being noticed. The latest onset of malignancy was 35 years after sarcoidosis had been confirmed. ${ }^{16}$

Cohen and Kurzrock define malignancy and sarcoidosis in three settings: sarcoidosis followed by a hematologic malignancy, sarcoidosis followed by a solid tumor and sarcoidosis as a paraneoplastic reaction due to malignancy. They also described sarcoid reaction (i.e. the development of noncaseating granulomas in patients who do not fulfil the criteria for systemic sarcoidosis) ${ }^{17}$, which is restricted to regional lymph nodes or the visceral organ of tumor origin, but rarely appears in the skin. ${ }^{18}$ Several studies evaluated a number of sarcoid reactions on a PET-CT scan before or after treatment of a malignancy. ${ }^{17,19-22}$

A few authors report malignancy first and then followed by the histologically proven sarcoidosis. ${ }^{12,13,23-25}$ Suen et al. reported of a reverse condition than Bricker, they called it a "syndrome of sarcoidosis following malignancy with or without chemotherapy". ${ }^{12}$ The malignancies reported were lymphoproliferative diseases ${ }^{13}$, breast ${ }^{12,17}$ and ovarian cancer ${ }^{12,25}$, Hodgkin lymphoma ${ }^{24}$,

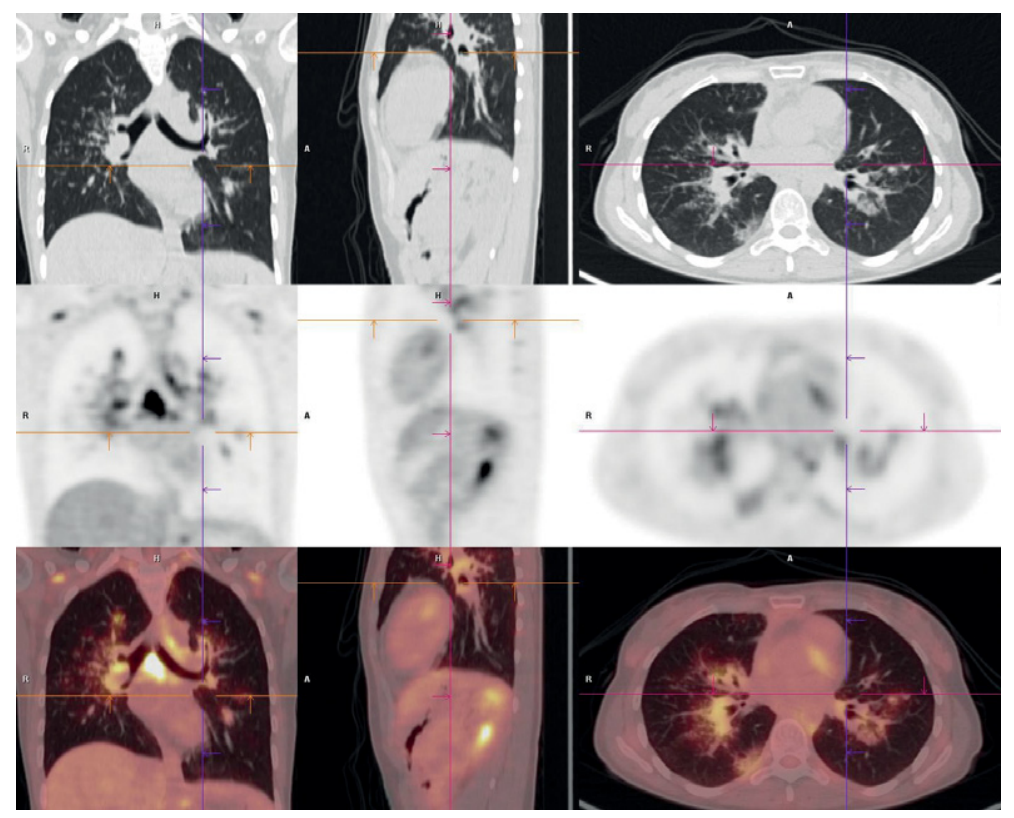

FIGURE 2. PET-CT patient 2: SUspicious relapse in hilar lymph nodes.

MALT lymphoma ${ }^{26}$, nonseminomatous malignant germ cell tumor, osteosarcoma and oral floor carcinoma. ${ }^{17}$ We found two separate articles describing two patients who underwent autologous stem cell transplantation and were later diagnosed with sarcoidosis, which was not present at the time of treatment of lymphoma. ${ }^{12,13}$ One was a 41 -year-old male with diffuse large B-cell lymphoma, who un-



FIGURE 3. PET-CT patient 3: SUspicious relapse in mediastinal lymph nodes. 
derwent chemotherapy and AuSCT and after few months complained of arthralgias with undulating fever and erythematous lesions of the skin, which were attributed to sarcoidosis. ${ }^{13}$ The other case was a 28-year-old male with T-cell lymphoma who underwent chemotherapy and allogeneic stem cell transplantation (AlSCT). After 15 months he complained of weight loss, cough and dyspnea. He was treated with steroids, yet developed severe pulmonary fibrosis and died 28 months after the onset of sarcoidosis. $^{12}$

The largest study by Ulaner et al. involved 251 patients after autologous or allogeneic stem cell transplantation treatment for lymphoma. In AlSCT group, 50 out of 107 patients had FDG-avid lesion after transplantation, 21 of which were histologically identified as benign - there were no specific data provided by the authors about the biopsies, benign changes were attributed to subclinical inflammation due to graft-versus-host-disease or graft-versus-lymphoma process. In AuSCT group, 65 out of 144 patients had FDG-lesion after transplantation on a PET-CT scan, $91 \%$ of them were demonstrated to be lymphoma by biopsy or follow-up imaging. ${ }^{2}$ Considering their subdivision of groups, our three patients would fit in the $9 \%$ of non-malignant changes after AuSCT, which are by their criteria a rare entity.

The pathogenesis of sarcoidosis after treatment of malignancy is still unknown and different theories and hypotheses were proposed. Merchant et al. suggested that sarcoidosis develops as a direct immunosuppressive effect of chemotherapy or due to the influence of a specific chemotherapy agent, bleomycin, which is known to have a relatively high lymph node, skin and lung tissue concentrations. ${ }^{24}$ Brincker, on the other hand, proposed that the formation of epithelioid-cell granulomas is driven by the antigenic factors derived from tumor cells, eliciting an immunological hypersensitivity reaction which results in granuloma formation. ${ }^{22}$ And finally, Kornacker et al. suggested that the development of sarcoidosis is a consequence of an underlying immunologic disturbance associated with the primary malignancy. ${ }^{13}$

\section{Conclusions}

The PET-CT is the recommended diagnostic tool for post-treatment assessment in some of the NHL - namely the FDG-avid ones. It gives us valuable information about the residual disease but it is not one hundred percent accurate. Our cases clearly demonstrate that the confirmation by biopsy of a residual mass or lymph node is needed as there are other pathologies that could mimic relapse and would lead us into the wrong direction of treatment. Sarcoidosis prior to malignancy is a known condition, though not very frequently observed. Sarcoidosis after treatment of malignancy is even less frequent but still possible. Biopsy is crucial.

\section{References}

1. Valeyre D, Prasse A, Nunes $H$, Uzunhan $Y$, Brillet PY, Müller-Quernheim J. Sarcoidosis. Lancet 2014; 383: 1155-67.

2. Ulaner GA, Lilienstein J, Gönen $\mathrm{M}$, Maragulia J, Moskowitz $\mathrm{CH}$, Zelenetz AD. False-Positive [18F]fluorodeoxyglucose-avid lymph nodes on positron emission tomography-computed tomography after allogeneic but not autologous stem-cell transplantation in patients with lymphoma. J Clin Oncol 2014; 32: 51-6.

3. Barrington $S F$, Mikhaeel NG, Kostakoglu L, Meignan M, Hutchings $M$, Mueller SP, et al. Role of imaging in the staging and response assessment of lymphoma: Consensus of the international conference on malignant lymphomas imaging working group. J Clin Oncol 2014; 32: 3048-58.

4. Maza S, Buchert R, Brenner W, Munz DL, Thiel E, Korfel A, et al. Brain and whole-body FDG-PET in diagnosis, treatment monitoring and long-term follow-up of primary CNS lymphoma. Radiol Oncol 2013; 47: 103-10.

5. Spaepen K, Stroobants S, Dupont P, Van Steenweghen S, Thomas J, Vandenberghe $P$, et al. Prognostic value of positron emission tomography (PET) with fluorine-18 fluorodeoxyglucose ([18F]FDG) after first-line chemotherapy in non-Hodgkin's lymphoma: is [18F]FDG-PET a valid alternative to conventional diagnostic methods? J Clin Oncol 2001; 19: 414-9.

6. Spagnolo P, Luppi F, Roversi P, Cerri S, Fabbri LM, Richeldi L. Sarcoidosis: challenging diagnostic aspects of an old disease. Am J Med 2012; 125: 118-25.

7. Brady B, Kamel D, Kiely J, Hennessy B. Dual diagnosis of sarcoidosis and lymphoma. Ir J Med Sci 2013; 182: 283-6.

8. Han HS, Escalón MP, Hsiao B, Serafini A, Lossos IS. High incidence of falsepositive PET scans in patients with aggressive non-Hodgkin's lymphoma treated with rituximab-containing regimens. Ann Oncol 2009; 20: 309-18.

9. Jerusalem G, Beguin Y, Fassotte MF, Najiar F, Paulus $\mathrm{P}$, Rigo $\mathrm{P}$, et al. Wholebody positron emission tomography using $18 \mathrm{~F}$-fluorodeoxyglucose for posttreatment evaluation in Hodgkin's disease and non-Hodgkin's lymphoma has higher diagnostic and prognostic value than classical computed tomography scan imaging. Blood 1999; 94: 429-33.

10. Naumann R, Vaic A, Beuthien-Baumann B, Bredow J, Kropp J, Kittner T, et al. Prognostic value of positron emission tomography in the evaluation of post-treatment residual mass in patients with Hodgkin's disease and nonHodgkin's lymphoma. Br J Haematol 2001; 115: 793-800.

11. Gawande RS, Khurana A, Messing S, Zhang D, Castaneda RT, Goldsby RE, et al. Differentiation of normal thymus from anterior mediastinal lymphoma and lymphoma recurrence at pediatric PET/CT. Radiology 2012; 262: 61322.

12. Suen JS, Forse MS, Hyland RH, Chan CK. The malignancy-sarcoidosis syndrome. Chest 1990; 98: 1300-2.

13. Kornacker M, Kraemer A, Leo E, Ho AD. Occurrence of sarcoidosis subsequent to chemotherapy for non-Hodgkin's lymphoma: report of two cases. Ann Hematol 2002; 81: 103-5.

14. Fishman JA. Approach to the immunocompromised patient with fever and pulmonary infiltrates. In: Marr KA, Thorner AR, editors. Uptodate. com. UpToDate; C 2013 [citated 2014 Oct 19]. Available from: http://www. uptodate.com/

15. Brincker $\mathrm{H}$, Wilbek $\mathrm{E}$. The incidence of malignant tumours in patients with respiratory sarcoidosis. Br J Cancer 1974; 29: 24-52.

16. Brincker H. The sarcoidosis-lymphoma-syndrome. Br J Cancer 1986; 54: 467-73. 
17. Inoue K, Goto R, Shimomura H, Fukuda H. FDG-PET/CT of sarcoidosis and sarcoid reactions following antineoplastic treatment. Springerplus 2013; 2: 113 .

18. Cohen PR, Kurzrock R. Sarcoidosis and malignancy. Clin Dermatol 2007; 25: 326-33.

19. Chowdhury FU, Sheerin F, Bradley KM, Gleeson FV. Sarcoid-like reaction to malignancy on whole-body integrated (18)F-FDG PET/CT: prevalence and disease pattern. Clin Radiol 2009; 64: 675-81.

20. Hunsaker AR, Munden RF, Pugatch RD, Mentzer SJ. Sarcoidlike reaction in patients with malignancy. Radiology 1996; 200: 255-61.

21. Hunt BM, Vallières $E$, Buduhan $G$, Aye R, Louie B. Sarcoidosis as a benign cause of lymphadenopathy in cancer patients. Am J Surg 2009; 197: 629-32.

22. Brincker H. Sarcoid reactions in malignant tumours. Cancer Treat Rev 1986; 13: $147-56$

23. Haran $M Z$, Feldberg E, Berrebi A. Lymphoma masking sarcoidosis. Leuk Lymphoma 2002; 43: 1709-10.

24. Merchant TE, Filippa DA, Yahalom J. Sarcoidosis following chemotherapy for Hodgkin's disease. Leuk Lymphoma 1994; 13: 339-47.

25. Kim MH, Lee K, Kim KU, Park HK, Lee MK, Suh DS. Sarcoidosis mimicking cancer metastasis following chemotherapy for ovarian cancer. Cancer Res Treat 2013; 45: 354-8.

26. Torchio M, Bottaro G, Bertolino G, Comolli G, Bello BD, Invernizzi R, et al. Late-onset sarcoidosis in a patient with gastric mucosa-associated lymphoid tissue non-Hodgkin lymphoma: A case report. Oncol Lett 2014; 8: 1299-301. 